\title{
Study of pretransitional behavior of laser-field-induced molecular alignment in isotropic nematic substances
}

\author{
George K. L. Wong and Y. R. Shen \\ Department of Physics, University of California \\ and Inorganic Materials Research Division, Lawrence Berkeley Laboratory, Berkeley, California 94720
}

(Received 15 April 1974)

\begin{abstract}
We have measured the optical Kerr effect and the intensity-dependent ellipse rotation in order to study the pretransitional behavior of field-induced molecular alignment in the isotropic nematic substances $p$-methoxy-benzylidene $p$ - $n$-butylaniline (MBBA) and $p$-ethoxy-benzylidene- $p$-butylaniline (EBBA). The results agree well with predictions of the Landau-de Gennes model. Both the orderparameter relaxation time and the steady-state field-induced birefringence show critical divergence towards the isotropic $\rightarrow$ nematic transition with a $\left(T-T^{*}\right)^{-1}$ temperature dependence. In the case of MBBA, our results are also consistent with the results from light scattering, but the method we use is perhaps more straightforward and accurate. The nonlinear refractive indices and other relevant parameters of the materials are derived from the experiment.
\end{abstract}

\section{INTRODUCTION}

We have shown recently by measuring the optical Kerr effect and the intensity-dependent ellipse rotation that the nematic compound $p$-methoxy-benzylidene $p$ - $n$-butylaniline (MBBA) in its isotropic phase has a large nonlinear refractive index and a pronounced pretransitional behavior. ${ }^{1}$ The large field-induced refractive index causes a moderately intense laser pulse to self-focus readily in such a medium ${ }^{2,3}$ and induces other nonlinear optical effects such as stimulated Raman and Brillouin scattering. ${ }^{4}$ On the other hand, measurements of the optical-field-induced refractive index and its pretransitional behavior yield directly information about the molecular orientational properties of $\mathrm{MBBA}^{1}$ and provides a stringent test on the Landau-de Gennes model. ${ }^{5}$ The same information can be obtained from light-scattering experiments, ${ }^{6}$ but the measurements are more complicated and less accurate. We have now extended our optical Kerr and ellipse rotation measurements to $p$ ethoxy-benzylidene-p-butylaniline (EBBA) which is homologous to MBBA. We have found similar results in EBBA as in MBBA. In particular, the results again agree well with the predictions of the Landau-de Gennes model. Here we would like to give a detailed account of our work on both MBBA and EBBA.

In Sec. II, we review briefly the theories behind our measurements. In Sec. III, we show our experimental arrangements and compare our results with the predictions of the Landau-de Gennes model. We discuss our results in Sec. IV and compare them with results obtained from other measurements.

\section{THEORETICAL BACKGROUND}

We first give a brief review on the theories of the optical Kerr effect and the ellipse-rotation effect. We then discuss these effects in connection with the pretransitional behavior of liquid crystalline materials in the isotropic phase.

\section{A. Optical-field-induced nonlinear refractive indices of an isotropic medium}

The optical susceptibility of a medium is, in general, a function of the applied optical fields. For a medium with inversion symmetry, the fieldinduced optical susceptibility in the lowest order can be written as ${ }^{7}$

$$
\delta \chi_{i j}=6 \chi_{i j k l}^{(3)}\left(\omega=\omega+\omega^{\prime}-\omega^{\prime}\right) E_{k}\left(\omega^{\prime}\right) E_{l}^{*}\left(\omega^{\prime}\right) .
$$

By symmetry, the third-order nonlinear susceptibility tensor $\chi_{i j k l}^{(3)}\left(\omega=\omega+\omega^{\prime}-\omega^{\prime}\right)$ of an isotropic medium has the following nonvanishing elements ${ }^{8}$ $(i, j=x, y, z)$ :

$$
\begin{aligned}
& \chi_{1111}^{(3)}=\chi_{i i i i}^{(3)}, \\
& \chi_{1122}^{(3)}=\chi_{i i j j}^{(3)}, \quad \chi_{1212}^{(3)}=\chi_{i j i j}^{(3)}, \\
& \chi_{1221}^{(3)}=\chi_{i j j i}^{(3)}, \\
& \chi_{1111}^{(3)}=\chi_{1212}^{(3)}+\chi_{1221}^{(3)}+\chi_{1122}^{(3)},
\end{aligned}
$$

If $\omega=\omega^{\prime}$, then $\chi_{1122}^{(3)}=\chi_{1212}^{(3)}$. The corresponding nonlinear polarization is ${ }^{7.9}$

$$
\begin{gathered}
P_{i}^{(3)}(\omega)=\sum_{j} 6\left[\chi_{1122}^{(3)}\left(\omega=\omega+\omega^{\prime}-\omega^{\prime}\right) E_{i}(\omega) E_{j}\left(\omega^{\prime}\right) E_{j}^{*}\left(\omega^{\prime}\right)+\chi_{1212}^{(3)}\left(\omega=\omega+\omega^{\prime}-\omega^{\prime}\right) E_{j}(\omega) E_{i}\left(\omega^{\prime}\right) E_{j}^{*}\left(\omega^{\prime}\right)\right. \\
\left.+\chi_{1221}^{(3)}\left(\omega=\omega+\omega^{\prime}-\omega^{\prime}\right) E_{j}(\omega) E_{j}\left(\omega^{\prime}\right) E_{i}^{*}\left(\omega^{\prime}\right)\right] .
\end{gathered}
$$


In general, the field-induced refractive index contains an electronic part due to field-induced deformation of the electron cloud around molecules and a nuclear part due to molecular reorientation and redistribution by the field. According to Owyoung et $a l .{ }^{10}$ we have for an isotropic medium

$$
\begin{aligned}
& \chi_{1221}^{(3)}\left(\omega=\omega+\omega^{\prime}-\omega^{\prime}\right)=\frac{1}{24}(\sigma+2 \beta), \\
& \chi_{1212}^{(3)}+\chi_{1221}^{(3)}=\frac{1}{12}(\sigma+\beta),
\end{aligned}
$$

where $\sigma$ and $\beta$ are contributions from the electronic part and the nuclear part, respectively. If $\sigma=0$, then $\chi_{1212}^{(3)}=0$ and $\chi_{1221}^{(3)}=\frac{1}{12} \beta$. If $\beta=0$, then $\chi_{1221}^{(3)}=\chi_{1212}^{(3)}=\frac{1}{24} \sigma$. We can determine $\sigma$ and $\beta$ or $\chi_{1212}^{(3)}$ and $\chi_{1221}^{(3)}$ by measuring both the optical Kerr effect and the ellipse rotation. ${ }^{11}$

We have assumed in the above discussion monochromatic fields. In practice, the strong optical field may be a pulse represented by the field components

$$
E_{i}\left(\omega^{\prime}, t\right)=\hat{i}^{*} \cdot \hat{e} \mathcal{E}(t) \exp \left(i \overrightarrow{\mathrm{k}}^{\prime} \cdot \overrightarrow{\mathrm{r}}-i \omega^{\prime} t\right),
$$

where $\mathcal{E}(t)$ is the amplitude function. The fieldinduced susceptibility becomes

$$
\begin{aligned}
\delta \chi_{i j}\left(\omega, \omega^{\prime} ; t\right)= & \int_{-\infty}^{t} \sum_{k, l} 6 \chi_{i j k l}^{(3)}\left(\omega, \omega^{\prime} ; t-t^{\prime}\right) \\
& \times|\mathcal{E}|^{2}\left(t^{\prime}\right) \hat{k}^{*} \cdot \hat{e} \hat{l} * \cdot \hat{e} .
\end{aligned}
$$

The electronic part and the nuclear part of $\chi_{i j k l}^{(3)}$ should of course have different functional dependence on time. The electronic part has a response time of the order of $10^{-15} \mathrm{sec}$, while the nuclear part can have a much slower response. Therefore, for ordinary laser pulses, we can regard the electronic response as instantaneous and write

$$
\begin{aligned}
& \chi_{1221}^{(3)}\left(\omega, \omega^{\prime} ; t-t^{\prime}\right) \\
& =\frac{1}{24}\left[\sigma\left(\omega, \omega^{\prime}\right) \delta\left(t-t^{\prime}\right)+2 \beta^{\prime}\left(\omega, \omega^{\prime} ; t-t^{\prime}\right],\right. \\
& \left(\chi_{1221}^{(3)}+\chi_{1212}^{(3)}\right)\left(\omega, \omega^{\prime} ; t-t^{\prime}\right) \\
& =\frac{1}{12}\left[\sigma\left(\omega, \omega^{\prime}\right) \delta\left(t-t^{\prime}\right)+\beta^{\prime}\left(\omega, \omega^{\prime} ; t-t\right)\right] .
\end{aligned}
$$

For molecular reorientation and redistribution governed by a diffusion equation, we expect the response function $\beta$ to have the form

$$
\beta^{\prime}\left(\omega, \omega^{\prime} ; t\right)=\left[\beta\left(\omega, \omega^{\prime}\right) / \tau\right] e^{-t / \tau},
$$

where $\beta\left(\omega, \omega^{\prime}\right)$ is the response function for an infinitely long pulse and $\tau$ is the relaxation time. We shall show Eq. (8) explicitly, later, for an isotropic liquid-crystalline medium.

\section{B. Optical Kerr effect}

In the presence of a strong linearly polarized optical beam, an isotropic medium shows linear birefringence. This induced linear birefringence is given by

$$
\delta n_{l}=\delta n_{\|}-\delta n_{\perp}
$$

where $\delta n_{\|, \perp}=(2 \pi / n) \delta \chi_{\|, \perp}$. From Eqs. (6)-(8), we find

$$
\begin{aligned}
\delta n_{l}\left(\omega, \omega^{\prime} ; t\right) & =(2 \pi / n) \int_{-\infty}^{t} 6\left(\chi_{1221}^{(3)}+\chi_{1212}^{(3)}\right)\left(\omega, \omega^{\prime} ; t-t^{\prime}\right)|\mathcal{E}|^{2}\left(t^{\prime}\right) d t^{\prime} \\
& =(\pi / n)\left[\sigma\left(\omega, \omega^{\prime}\right)|\mathcal{E}|^{2}(t)+\int_{-\infty}^{t} \beta^{\prime}\left(\omega, \omega^{\prime} ; t-t^{\prime}\right)|\mathcal{E}|^{2}\left(t^{\prime}\right) d t^{\prime}\right] \\
& =(\pi / n)\left[\sigma\left(\omega, \omega^{\prime}\right)|\mathcal{E}|^{2}(t)+\left[\beta\left(\omega, \omega^{\prime}\right) / \tau\right] \int_{-\infty}^{t} e^{-\left(t-t^{\prime}\right) / \tau}|\mathcal{E}|^{2}\left(t^{\prime}\right) d t^{\prime}\right] .
\end{aligned}
$$

If the variation of $|\mathcal{E}|^{2}(t)$ is negligible in a time $\tau$, then Eq. (10) reduces to the usual expression

$$
\begin{aligned}
\delta n_{l}\left(\omega, \omega^{\prime}, t\right)= & (\pi / n)(\sigma+\beta)\left(\omega, \omega^{\prime}\right)|\mathcal{E}|^{2}(t) . \\
& \text { C. Ellipse rotation }{ }^{7,9}
\end{aligned}
$$

The effect of the field-induced refractive index on the propagation of an elliptically polarized beam is most easily understood by transforming $P_{i}^{(3)}(\omega)$ and $E_{i}(\omega)$ into the circular coordinates $\hat{e}_{+}=(\hat{x}+i \hat{y}) / \sqrt{2}$ and $\hat{e}_{-}=(\hat{x}-i \hat{y}) / \sqrt{2}$. One finds from Eq. (3) for $\omega=\omega^{\prime}$

$$
P_{ \pm}^{(3)}(\omega)=6\left[\chi_{1212}^{(3)}\left|E_{ \pm}\right|^{2}+\left(\chi_{1212}^{(3)}+\chi_{1221}^{(3)}\right)\left|E_{\mp}\right|^{2}\right] E_{ \pm}(\omega)
$$

where $E_{ \pm}=\left(E_{x} \pm i E_{y}\right) / \sqrt{2}$ and $P_{ \pm}=\left(P_{x} \pm i P_{y}\right) / \sqrt{2}$. The induced circular birefringence seen by the beam is

$$
\delta n_{c}=(2 \pi / n)\left(\delta \chi_{-}-\delta \chi_{+}\right) .
$$

From Eqs. (6)-(8), and (12), we obtain 


$$
\begin{aligned}
\delta n_{c}(\omega, \omega ; t) & =\frac{2 \pi}{n} \int_{-\infty}^{t} 6 \chi_{1221}^{(3)}\left(\omega, \omega ; t-t^{\prime}\right)|\mathcal{E}|^{2}\left(t^{\prime}\right) d t^{\prime}\left(\left.\hat{e}_{+}^{*} \cdot \hat{e}\right|^{2}-\left|\hat{e}_{-}^{*} \cdot \hat{e}\right|^{2}\right) \\
& =\frac{\pi}{2 n}\left(\sigma(\omega, \omega)|\mathcal{E}|^{2}(t)+\frac{2 \beta(\omega, \omega)}{\tau} \int_{-\infty}^{t} e^{-\left(t-t^{\prime}\right) / \tau}|\mathcal{E}|^{2}\left(t^{\prime}\right) d t^{\prime}\right)\left(\left|\hat{e}_{+}^{*} \cdot \hat{e}\right|^{2}-|\hat{e} \underset{-}{*} \hat{e}|^{2}\right) .
\end{aligned}
$$

In the quasi-steady-state, it reduces to

$$
\begin{aligned}
\delta n_{c}= & (\pi / 2 n)(\sigma+2 \beta)(\omega, \omega)|\mathcal{E}|^{2}(t) \\
& \times\left(\left|\hat{e}_{+}^{*} \cdot \hat{e}\right|^{2}-\left|\hat{e}_{-}^{*} \cdot \hat{e}\right|^{2}\right) .
\end{aligned}
$$

As the beam traverses the medium, this induced circular birefringence leads to a rotation $\theta$ of the polarization ellipse with

$$
\frac{d \theta}{d z}=(\omega / 2 c) \delta n_{c}
$$

D. Landau-de Gennes model for the pretransitional behavior of liquid crystalline substances

de Gennes ${ }^{5}$ has successfully applied Landau's theory of second-order phase transition to describe the isotropic $\rightarrow$ mesomorphic pretransitional behavior of liquid crystalline materials. We briefly review the theory here. We shall limit our discussion to nematic substances only.

Let $Q_{i j}$ be the macroscopic tensor order parameter which describes the ordering in molecular orientation. As pointed out by de Gennes, ${ }^{5}$ any tensorial property of the medium can be used to define $Q_{i j}$. For example, we can define

$$
\chi_{i j}=\bar{\chi}_{i j} \delta_{i j}+\frac{2}{3} \Delta \chi Q_{i j}
$$

where $\bar{\chi}=\sum_{i} \frac{1}{3} \chi_{i i}$ and $\Delta \chi$ is the anisotropy in $\chi_{i j}$ when all molecules are perfectly aligned in one direction. The free energy per unit volume in the isotropic phase is given by

$$
\begin{aligned}
& F=F_{0}+\frac{1}{2} A Q_{i j} Q_{j i}-\frac{1}{4} \chi_{i j} E_{i}^{*} E_{j}, \\
& A=a(T-T *)
\end{aligned}
$$

where $a$ and $T^{*}$ are constants. We have neglected in the above expression higher-order terms of $Q_{i j}$ and the spatial dependence of $Q_{i j}$. The corresponding dynamic equation for $Q_{i j}$ is

$$
\begin{aligned}
& \frac{\nu \partial Q_{i j}}{\partial t}+A Q_{i j}=f_{i j}, \\
& f_{i j}(t)=\frac{1}{6} \Delta \chi\left(E_{i}^{*} E_{j}-\frac{1}{3}|E|^{2} \delta_{i j}\right)(t),
\end{aligned}
$$

where $\nu$ is a viscosity coefficient. The solution of the above equation is

$$
Q_{i j}(t)=\int_{-\infty}^{t}\left[f_{i j}\left(t^{\prime}\right) / \nu\right] e^{-\left(t-t^{\prime}\right) / \tau} d t^{\prime},
$$

where

$$
\tau=\nu / A=\nu / a\left(T-T^{*}\right) .
$$

From Eq. (17), we find that the linear birefringence induced by a strong linearly polarized field along $\hat{i}$ is

$$
\begin{aligned}
& \delta n_{l}=(2 \pi / n) \frac{2}{3} \Delta \chi\left(Q_{i i}-Q_{j j}\right)=(2 \pi / n) \Delta \chi Q_{i i}, \\
& Q_{i i}=\frac{1}{9}\left(\Delta_{\chi} / \nu\right) \int_{-\infty}^{t}|\mathcal{E}|^{2}\left(t^{\prime}\right) e^{-\left(t-t^{\prime}\right) / \tau} d t^{\prime} .
\end{aligned}
$$

If $|\mathcal{E}|^{2}(t)$ is a pulse shorter than or comparable with $\tau$, then at sufficiently large time $t$, both $Q_{i i}$ and $\delta n_{l}$ will decrease exponentially with a time constant $\tau$. We have considered here only the nuclear contribution to the induced refractive index. Then, comparing Eq. (22) with Eq. (10c), with $\sigma=0$, we find

$$
\beta\left(\omega, \omega^{\prime}\right)=2(\Delta \chi)^{2} / 9 A=2(\Delta \chi)^{2} / 9 a\left(T-T^{*}\right) .
$$

Thus, by deducing $\tau$ and $\beta\left(\omega, \omega^{\prime}\right)$ from experimental results as a function of temperature, we can determine $\nu / a$ and $(\Delta \chi)^{2} / a$.

\section{EXPERIMENTS AND RESULTS}

\section{A. Sample preparation}

We made measurements on the two homologous nematic compounds MBBA and EBBA. The samples were purchased from Eastman Kodak and Vari-Light Corporation. They were used without further purification. The sample was placed in a glass cell of $4 \mathrm{~cm}$ long with end windows free of strain birefringence. The cell was pumped under vacuum for several hours and then sealed under 1-atm pressure of $\mathrm{N}_{2}$ gas. The transition temperatures of the samples prepared this way showed no change over a period of months. The cell was then placed in a closely fitted copper block and thermally controlled by a Yellow-Spring thermo control unit. The temperature along the cell was found to be uniformly stabilized to within $\pm 0.03^{\circ} \mathrm{C}$. The clearing temperature $T_{K}$ of our samples are $42.5^{\circ} \mathrm{C}$ and $78.5^{\circ} \mathrm{C}$ for $\mathrm{MBBA}$ and $\mathrm{EBBA}$, respectively.

$$
\begin{gathered}
\text { B. Measurements of orientational relaxation } \\
\text { times }
\end{gathered}
$$

We used a single-mode ruby laser $Q$ switched by cryptocyanine in methanol. The single spatial mode of the laser beam was achieved by placing a $0.8-\mathrm{mm}$ pinhole inside the cavity. The output 


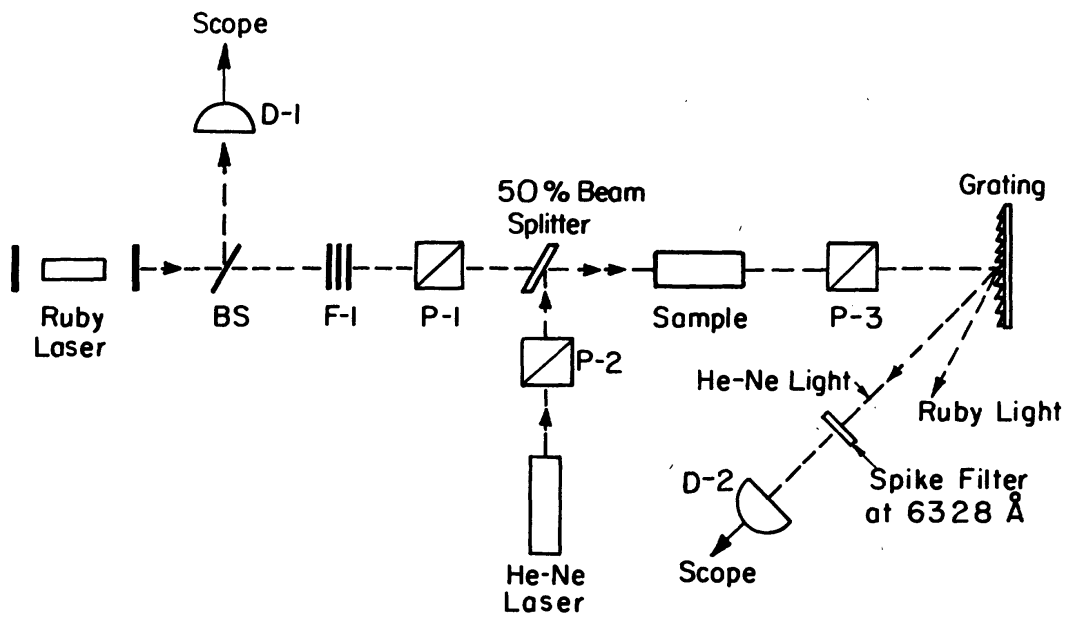

FIG. 1. Experimental arrangement for observing molecular orientational relaxation times in nematic liquid crystals. BS, beam splitter; P-1, P-2, P-3 linear polarizers; $\mathrm{D}-1$, ITT F4018 fast photodiode; D-2, RCA photomultiplier 7102; F-1, neutral-density stacks.

pulse width was about 10 nsec (full width at halfmaximum) and the maximum peak power was about $50 \mathrm{~kW}$.

The experimental arrangement for measuring relaxation times is shown in Fig. 1. The finite ordering was induced by the linearly polarized laser beam. The subsequent time variation of the ordering parameter was probed by a $40-\mathrm{mW}$ $\mathrm{cw} \mathrm{He}-\mathrm{Ne}$ gas laser. The polarization of the $\mathrm{He}-\mathrm{Ne}$ laser beam was at $45^{\circ}$ to that of the ruby beam. The polarizer P-3 was crossed with the polarizer P-2 so that signal could reach the photomultiplier only when the medium was birefringent resulting from induced ordering in the sample. Both the ruby and the He-Ne laser beams were telescoped down to $\sim 0.5 \mathrm{~mm}$ inside the sample cell. The He-Ne laser power going into the sample was about $10 \mathrm{~mW}$ and the peak power of the

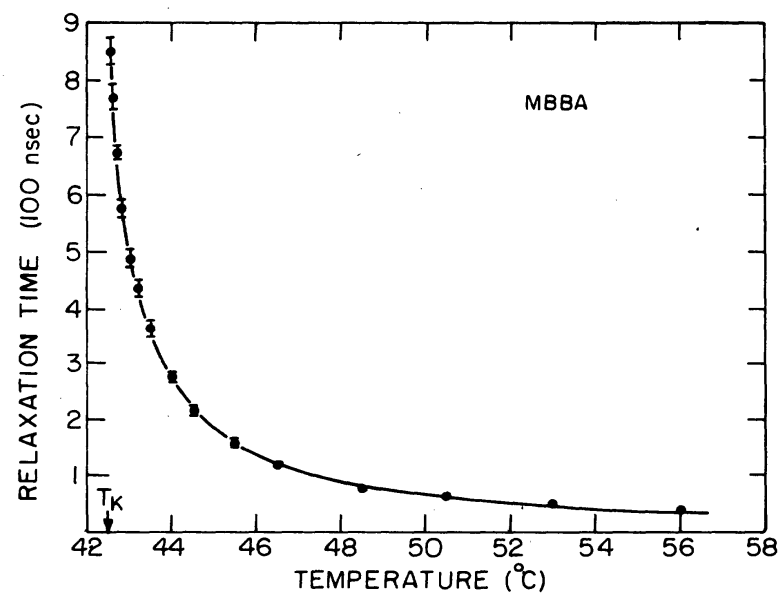

FIG. 2. Relaxation time $\tau$ of the order parameter as a function of temperature for MBBA. The solid curve is the theoretical curve described in the text. The dots are the experimental data points. ruby laser pulse was about $10 \mathrm{~kW}$.

For this arrangement, the signal at the photomultiplier was proportional to $\sin ^{2}\left(K \delta n_{l}\right)$, where $K$ is a constant and $\delta n_{l}$ is the linear birefringence at the $\mathrm{He}-\mathrm{Ne}$ frequency. In our experiments, $K \delta n_{l} \ll 1$ and hence the photomultiplier signal was proportional to $\left(\delta n_{l}\right)^{2}$. Thus, if $\delta n_{l}$ or the order parameter decayed as $e^{-t / \tau}$, the signal would decay as $e^{-2 t / \tau}$. We found that our measured signals always had perfect exponential tails from which we then calculated the orientational relaxation times $\tau$. In Figs. 2 and 3, we present our experimental data of $\tau$ as a function of temperature for MBBA and EBBA. Both curves show clear divergence as $T$ approaches the transition temperature $T_{K}$. They agree very well with the theoretical curves given by $\tau=\nu / a\left(T-T^{*}\right)$ in Eq. (21) if we assume $\nu=\nu_{0} e^{W / T}$ with $W=2800^{\circ} \mathrm{K}$ as suggested

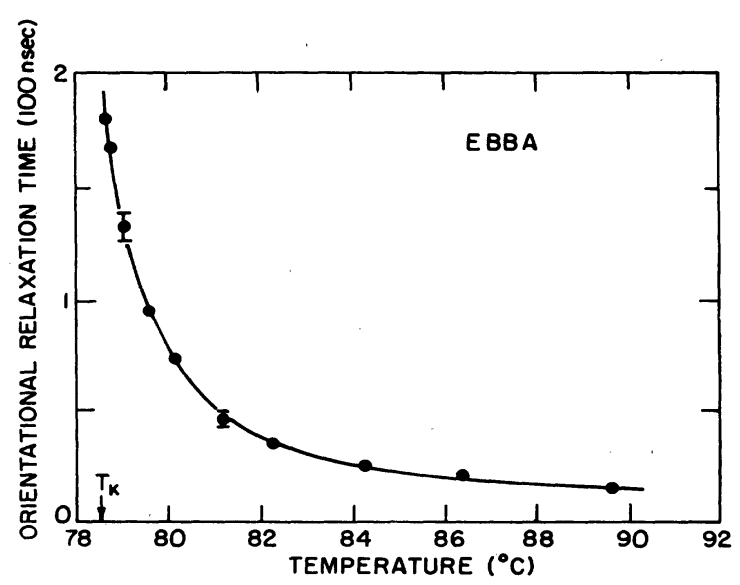

FIG. 3. Relaxation time $\tau$ of the order parameter as a function of temperature for EBBA. The solid curve is the theoretical curve described in the text. The dots are the experimental points. 
TABLE I. Results of optical Kerr, ellipse-rotation, and orientational relaxation-time measurements on MBBA and EBBA.

\begin{tabular}{|c|c|c|c|c|c|c|c|c|}
\hline Material & $\begin{array}{c}\sigma \\
\text { (esu) }\end{array}$ & $\begin{array}{c}\beta \\
\text { (esu) }\end{array}$ & $\begin{array}{c}\chi_{1122}(\omega, \omega)=\chi_{1212}(\omega, \omega) \\
(\mathrm{esu})\end{array}$ & $\begin{array}{l}\chi_{1221}(\omega, \omega) \\
\quad(\mathrm{esu})\end{array}$ & $\begin{array}{c}B \\
\text { (esu) }\end{array}$ & $\begin{array}{c}\nu / a \\
\left(\sec ^{\circ} \mathrm{K}\right)\end{array}$ & $\begin{array}{c}\frac{(\Delta \chi)^{2}}{a} \\
\left(\mathrm{erg}^{-1} \mathrm{~cm}^{3}{ }^{\circ} \mathrm{K}\right)\end{array}$ & $\begin{array}{c}T^{*} \\
\left(\mathrm{~K}^{\circ}\right)\end{array}$ \\
\hline MBBA & $<0.01 \beta$ & $\frac{2.7 \times 10^{-9}}{T-T^{*}}$ & $<0.01 \chi_{1221}(\omega, \omega)$ & $\frac{2.2 \times 10^{-10}}{T-T^{*}}$ & $\frac{1.5 \times 10^{-4}}{T-T^{*}}$ & $9.1 \times 10^{-11} e^{2800 / T}$ & $1.1 \times 10^{-8}$ & 314.7 \\
\hline EBBA & $<0.01 \beta$ & $\frac{1.5 \times 10^{-9}}{T-T^{*}}$ & $<0.01 \chi_{1221}(\omega, \omega)$ & $\frac{1.3 \times 10^{-10}}{T-T^{*}}$ & $\frac{8.6 \times 10^{-5}}{T-T^{*}}$ & $7.0 \times 10^{-11} e^{2800 / T}$ & $6.4 \times 10^{-9}$ & 350.6 \\
\hline
\end{tabular}

by Stinson and Litster. ${ }^{6}$ The values of $\nu_{0} / a$ and $T *$ deduced from the fit for MBBA and EBBA are given in Table I. For MBBA, the relaxation time varies from $\sim 40 \mathrm{nsec}$ at temperature far above the phase transition to $>800 \mathrm{nsec}$ near the transition. The relaxation time for EBBA is considerably shorter. It varies from about $13 \mathrm{nsec}$ to $\sim 170$ nsec. The results for MBBA are in good agreement with those obtained from light scattering by Stinson and Litster. ${ }^{6}$ However, we believe our measurements are more straightforward and accurate, especially when $\tau$ is long.

\section{Measurements of intensity-dependent ellipse rotation}

In Fig. 4, we show our experimental arrangement for ellipse-rotation measurements which was similar to that used by Owyoung et al. ${ }^{10}$ The Fresnel rhomb R-1 was used to produce a laser beam of desired ellipticity. The single-mode beam was focused into the sample by a $15-\mathrm{cm}$ lens L-1 so that the focus was at the center of the sample cell. The beam was then recollimated by lens L-2. The second Fresnel rhomb R-2 and the Glan polarizers were oriented in such a way that in the absence of ellipse rotation, the output beam from the Fresnel rhomb was linearly polarized, a maximum "transmitted" signal was directed into $\mathrm{D}-3$, and a minimum "nulled" signal into D-2. The purpose of D-3 was to monitor any nonlinear loss or change in the spatial profile of the laser beam. Neutral density filter stack F-1 was used to vary the input power. Laser power less than $1 \mathrm{~kW}$ was used in the experiment. If the focusing of the beam is weak enough so that self-focusing is absent, then geometric optics is a good approximation to describe the focused beam. Under such condition, one can show ${ }^{10}$ that for a single-mode beam with a Gaussian profile, the signal $S$ at D-2 with respect to the input laser power $P(t)$ is given by

$$
\begin{aligned}
& S(t) / P(t)=C(\sin 2 \phi)^{2} \theta_{\mathrm{av}}^{2}(t), \\
& \theta_{\mathrm{av}}=\frac{2 \pi^{2} \omega^{2}}{n c^{3}} \cos 2 \phi(\sigma P(t)+(2 \beta / \tau) \\
& \left.\quad \times \int_{-\infty}^{t} e^{-\left(t-t^{\prime}\right) / \tau} P\left(t^{\prime}\right) d t^{\prime}\right),
\end{aligned}
$$

where $C$ is a constant, $\tan \phi=\left|E_{+} / E_{-}\right|$, and we have assumed $\theta_{\mathrm{av}} \ll 1$. Note that the above result is independent of the focusing geometry and sample length.

In our experiments, we confined ourselves to low enough power so that $\theta \ll 1$. We also chose $\phi=22.5^{\circ}$. Since we can approximate our laser pulse well by a Gaussian pulse $P=P_{0} e^{-b^{2} t^{2}}$, we have from Eq. (24)

$$
S(0) / P_{0}=C\left(\pi^{2} \omega^{2} / n c^{3}\right)^{2}(\sigma+2 \beta g)^{2} P_{0}^{2},
$$

where $S(0)$ is the signal at the peak of the input pulse and

$$
g=(1 / b \tau) e^{1 / 4 b^{2} \tau^{2}}[1-\operatorname{erf}(1 / 2 b \tau)] .
$$

Because of the fluctuations of the ordering parameter, a nematic liquid crystal in its isotropic phase has a non-negligible scattering loss coefficient $^{11} \gamma$ which varies with temperature as ${ }^{12}$ $\gamma=\alpha /\left(T-T^{*}\right)$. Since the ellipse rotation occurred essentially within the focal volume which was lo-

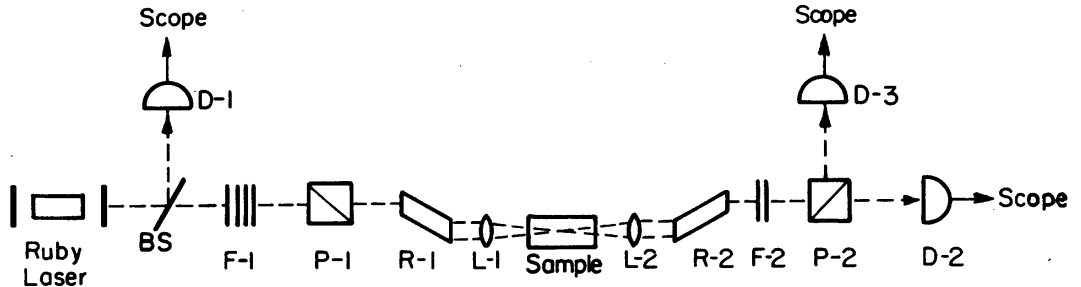

FIG. 4. Experimental arrangement for observing ellipse-rotation effect. $\mathrm{P}-1, \mathrm{P}-2$, Glan polarizers; $\mathrm{R}-1, \mathrm{R}-2$, fresnel rhombs; L-1, L-2, 15-cm lenses; F-1, F-2, neutral-density stacks; D-1, D-2, D-3 ITT fast photodiodes. 
TABLE $\Pi$. Values of $\nu$ and $a$ deduced from the experimental results shown in Table I.

\begin{tabular}{ccc}
\hline \hline Material & $\begin{array}{c}\nu \\
\text { (poise) }\end{array}$ & $\begin{array}{c}a \\
\left(\mathrm{erg} \mathrm{cm}^{-3}{ }^{\circ} \mathrm{K}^{-1}\right)\end{array}$ \\
\hline MBBA & $5.3 \times 10^{-5} e^{2800 / T}$ & $5.8 \times 10^{5}$ \\
EBBA & $7 \times 10^{-5} e^{2800 / T}$ & $1.0 \times 10^{6}$ \\
\hline \hline
\end{tabular}

cated at the center of the sample, the effect of scattering loss could be accounted for by rewriting Eq. (26) as

$$
\frac{S(0)}{P_{0} e^{-\gamma l}}=C\left(\frac{\pi^{2} \omega^{2}}{n c^{3}}\right)^{2}(\sigma+2 \beta g)^{2}\left(P_{0} e^{-\gamma l / 2}\right)^{2}
$$

or

$$
\frac{S(0)}{P_{0}}=C e^{-2 \gamma l}\left(\frac{\pi^{2} \omega^{2}}{n c^{3}}\right)^{2}(\sigma+2 \beta g)^{2} P_{0}^{2} .
$$

The scattering loss $e^{-\gamma l}$ was measured at each temperature. Thus, by plotting $S(0) / P_{0}$ vs $P_{0}^{2}$, we obtained $\sigma+2 \beta g$ as a function of temperature. To arrive at the absolute value, we used $\mathrm{CS}_{2}$ as a standard of calibration. As we shall show later, the comparison of $\sigma+2 \beta g$ with $\sigma+\beta g$ obtained from the optical Kerr effect indicates that $\sigma \ll \beta g$. Since we had measured the orientational relaxation time $\tau$ we could calculate $g$ and deduce $\beta$ from our measurements of $\sigma+2 \beta g$. The results for $2 \beta$ are shown in Figs. 5 and 6 as a function of temperature for MBBA and EBBA, respectively. We shall compare our results with the predictions of the

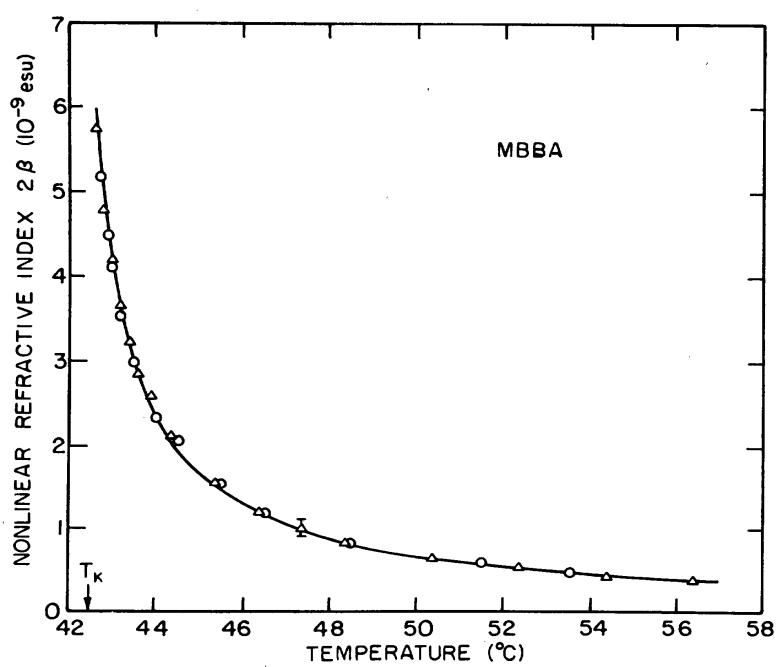

FIG. 5. Nonlinear refractive index $2 \beta$ as a function of temperature for MBBA. $\triangle$ (open triangles) are experimental data obtained from measurements of optical Kerr effect, $O$ (open circles) are experimental data obtained from measurements of ellipse-rotation effect. The solid curve is the theoretical curve given by $\left(5.4 \times 10^{-9}\right) /$ $\left(T-T^{*}\right)$ esu with $T^{*}=314.7^{\circ} \mathrm{K}$.
Landau-de Gennes model after the discussion of the optical Kerr measurements.

\section{Measurements of optical Kerr effect}

Our experimental arrangement is shown in Fig. 7. A linearly polarized ruby laser beam was sent through the sample to induce birefringence. The same beam after having its polarization direction rotated $45^{\circ}$ was attenuated and sent back through the sample to probe the birefringence. The analyzer P-3 was oriented in such a way that in the absence of birefringence, no signal could reach detector D-3. Maximum powers of the inducing beam and the probing beam were about $10 \mathrm{~kW}$ and $10 \mathrm{~W}$, respectively. Neglecting the delay of the returning probing beam $\left(<\frac{1}{2} \mathrm{nsec}\right)$ but taking into account the scattering loss, the signal $S(t)$ detected at D-3 should be related to the input power $P(t)$ by

$$
S(t)=\kappa P(t) e^{-2 \gamma l} \sin ^{2}\left[\frac{1}{2}\left(\frac{\omega}{c}\right) \frac{1-e^{\gamma l}}{\gamma} \delta n_{l}(\omega, \omega ; t)\right],
$$

where $\kappa P(t)$ is the probing beam power, and $\delta n_{l}(\omega, \omega ; t)$ is given by Eq. (10c). Since in our experiment, $(\omega / c) l \delta n_{l} \ll 1$ and $P=P_{0} e^{-b^{2} t^{2}}$, Eq. (29) becomes

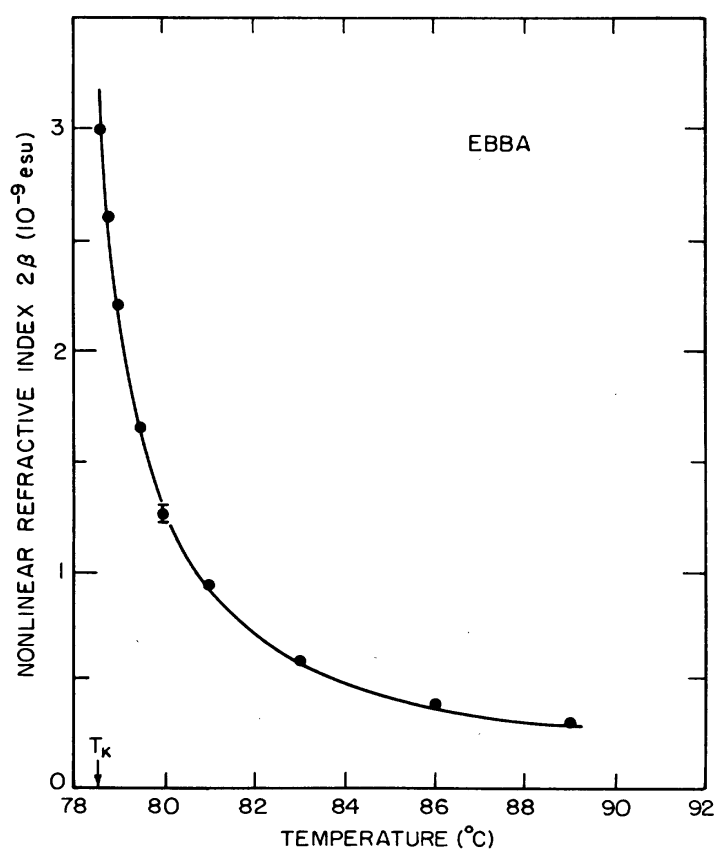

FIG. 6. Nonlinear refractive index $2 \beta$ as a function of temperature for EBBA. The dots are experimental data points obtained from measurements of ellipse-rotation effect. The solid curve is the theoretical curve given by $\left(3.1 \times 10^{-9}\right) /\left(T-T^{*}\right)$ esu with $T^{*}=350.6^{\circ} \mathrm{K}$. 


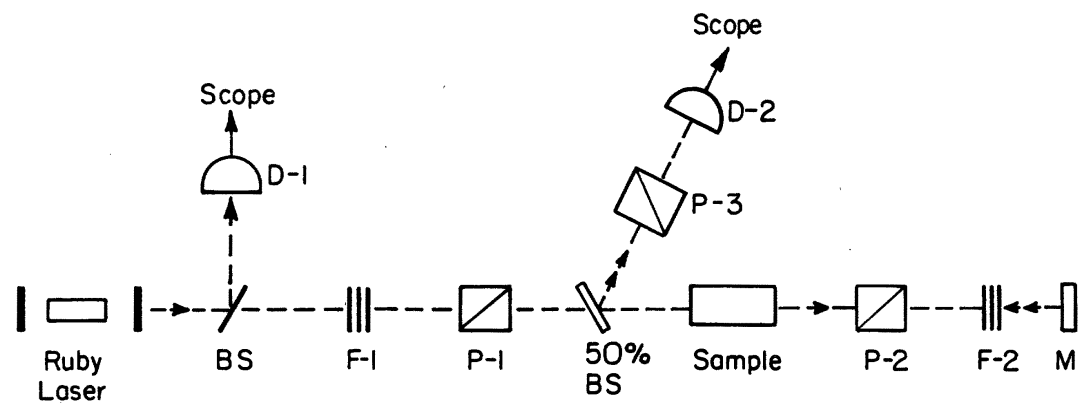

where $S(0)$ is the signal obtained at the peak of the laser pulse, $g$ is given by Eq. (27), and $\kappa^{\prime}$ is a constant proportional to $k$.

We obtained $\sigma+\beta g$ by plotting $S(0) / P_{0}$ vs $P_{0}^{2}$. Absolute values of $\sigma+\beta g$ were arrived at by using $\mathrm{CS}_{2}$ as a standard of calibration. By comparing $\sigma+\beta g$ from measurements of optical Kerr effect and $\sigma+2 \beta g$ from measurements of ellipse rotation, we concluded that $\sigma \ll \beta g$. Again, knowing $g$, we could deduce $\beta$ from the measured $\sigma+\beta g$. Results for MBBA are shown in Fig. 5. We see that the values of $2 \beta$ obtained from both measurements agree very well and show clear divergence as the temperature approaches $T_{K}$. The solid curve is calculated from

$$
\beta=2(\Delta \chi)^{2} / 9 a\left(T-T^{*}\right),
$$

given in Eq. (23) with

$$
2(\Delta \chi)^{2} / 9 a=2.7 \times 10^{-9} \mathrm{esu} \text {. }
$$

The corresponding optical Kerr constant $B$, given by $\omega \beta / n c$ is

$$
B=1.5 \times 10^{-4} /\left(T-T^{*}\right) \text { esu , }
$$

at $\lambda=6943 \AA$ for MBBA. Our data on EBBA also agree well with the theoretical curve calculated from Eq. (23) with

$$
2(\Delta \chi)^{2} / 9 a=1.6 \times 10^{-9} \mathrm{esu}
$$

as shown in Fig. 6. The corresponding optical Kerr constant is

$$
B=8.6 \times 10^{-5} /(T-T *) \text { esu. }
$$

\section{DISCUSSION}

We have seen in Sec. III that the temperature dependence of the orientational relaxation time and the nonlinear refractive index are in good agreement with the predictions of Landau-de Gennes model. We present in Table I the various

FIG. 7. Experimental arrangement for observing optical Kerr effect. BS, beam splitter; F-1, F-2, neutral-density stacks; $\mathrm{P}-1$, P-2, P-3, Glan polarizers; $M$, mirror; D-1, D-2; fast ITT F4108 photodiales. material coefficients we have deduced from our measurements for MBBA and EBBA. We can now compare our results with those obtained from light scattering. ${ }^{11}$

In Sec. II, we showed that by measuring the orientational relaxation times, we can obtain $\nu / a$ while by measuring the field-induced refractive indices, we can get $(\Delta \chi)^{2} / a$. In order to determine $a, \nu$, and $\Delta \chi$ separately, we need one more independent measurement. It happens that the value of $\Delta \chi$ for MBBA at $6328 \AA$ has been measured $^{13}$ to be $8.7 \times 10^{-2}$ in cgs units. The corresponding $\Delta \chi$ at $6943 \AA$ can be obtained in the following way. The temperature-dependent scattering loss coefficient $\alpha /\left(T-T^{*}\right)$ is proportional to $\omega^{4}(\Delta \chi)^{2} / a\left(T-T^{*}\right) .^{12}$ We have measured the scattering loss and found $\alpha$ to be $0.17 \mathrm{~cm}^{-1}{ }^{\circ} \mathrm{K}$ at $6328 \AA$ and $0.1 \mathrm{~cm}^{-1}{ }^{\circ} \mathrm{K}$ at $6943 \AA$ for MBBA. We then obtain $\Delta \chi(6943 \AA)=0.92 \times \Delta \chi(6328 \AA)=8 \times 10^{-2}$ in cgs units. With this value for $\Delta \chi$, we obtain $a_{\mathrm{MBBA}}=5.8 \times 10^{5} \mathrm{erg} \mathrm{cm}^{-3}{ }^{\circ} \mathrm{K}^{-1}$ and $\nu_{\mathrm{MBBA}}=5.3$ $\times 10^{-5} e^{2800 / T}$ poise for MBBA. Because of the uncertainties in $\Delta \chi$ and in our measurements, these values for $a$ and $\nu$ could have an uncertainty of $30 \%$. More accurate measurement on $\Delta \chi$ would reduce the uncertainty substantially. Our values of $a_{\mathrm{MBBA}}$ and $\nu_{\mathrm{MBBA}}$ are in good agreement with the

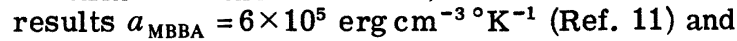
$\nu_{\text {MBBA }}=4 \times 10^{-5} e^{2800 / T}$ poise ${ }^{6,11}$ obtained from lightscattering experiments. For EBBA, $\Delta \chi$ has not been measured. However, since the molecular structure of MBBA and EBBA are rather similar (Fig. 8) and since the dominant contribution to $\Delta \chi$ should come from the benzene rings, it is reasonable to assume that $\triangle \chi$ has approximately the same value for MBBA and EBBA. Then, our experimen-

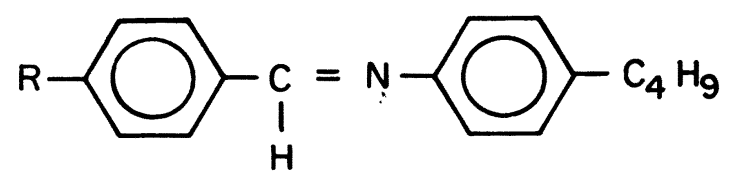

FIG. 8. Chemical structures for MBBA and EBBA. $R=\mathrm{CH}_{3}$ for MBBA and $R=\mathrm{C}_{2} \mathrm{H}_{5}$ for EBBA. 
tal results give $a_{\mathrm{EBBA}}=1 \times 10^{6} \mathrm{erg} \mathrm{cm}^{-3}{ }^{\circ} \mathrm{K}^{-1}$ and $\nu_{\text {EBBA }}=7 \times 10^{-5} e^{2800 / T}$ poise. With no light-scattering data on EBBA available, comparison of the results obtained from the two different methods is not possible at this time.

We notice that at a given temperature, the viscosity coefficient $\nu$ of EBBA is slightly larger than that of MBBA. This is expected because the molecular structure of EBBA is slightly longer than that of MBBA. The fact that EBBA has an appreciably shorter orientational relaxation time near the phase transition than MBBA is due to its higher clearing temperature. The mean-field parameters $a$ of the two materials have a ratio $a_{\mathrm{EBBA}} / a_{\mathrm{MBBA}}=1.7$. This seems to suggest that the additional $\mathrm{CH}_{3}$ group on EBBA has an effect of increasing the intermolecular interaction. With the latent heat given by $\frac{3}{4} a Q_{K}^{2} T_{K}$ at $T=T_{K},{ }^{11}$ this would predict an appreciably larger latent heat for EBBA.

Recently, Prost and Lalanne ${ }^{14}$ have also performed optical Kerr measurements on MBBA. Their results are different from ours and are also in disagreement with the predictions of the meanfield theory and with the results obtained from the light-scattering experiment. However, a neodymium-glass laser, $Q$ switched by rotating prism, was used in their experiments. It is well known that such a laser often produces a multimode laser beam with complicated spatial and temporal structure. Since knowledge of the temporal structure of the input pulse is important in their data analysis, this could yield considerable uncertainty in their results.

Finally, we should mention that the results presented in this paper were obtained with laser power well below the self-focusing threshold. The phenomenon became more complicated after self..focusing occurred. Investigation of the cause of this complication is currently in progress.

\section{ACKNOWLEDGMENT}

This work was supported by the U. S. Atomic Energy Commission.
${ }^{1}$ George K. L. Wong and Y. R. Shen, Phys. Rev. Lett. 30, 895 (1973).

${ }^{2}$ George K. L. Wong and Y. R. Shen, Phys. Rev. Lett. 32,527 (1974).

${ }^{3}$ D. V. G. L. N. Rao and S. Jayaraman, Appl. Phys. Lett. 23, 539 (1973).

${ }^{4}$ D. V. G. L. N. Rao and D. K. Agrawal, Phys. Lett. A 37,383 (1971).

${ }^{5}$ P. G. de Gennes, Phys. Lett. A 30,454 (1969) and Mol. Cryst. Liq. Cryst. 12, 193 (1971).

${ }^{6}$ T. W. Stinson III and J. D. Litster, Phys. Rev. Lett. $\underline{25}$, 503 (1970).

${ }^{7}$ P. D. Maker and R. W. Terhune, Phys. Rev. 137, A801 (1965).

${ }^{8}$ P. N. Butcher, Nonlinear Optical Phenomena, University Engineering Publications, Columbus, Ohio, 1965.

${ }^{9}$ C. C. Wang, Phys. Rev. 152, 149 (1965).

${ }^{10}$ A. Owyoung, R. W. Hellwarth, and N. George, Phys.
Rev. B $\underline{4}, 2342$ (1971); $\underline{5}, 628$ (1972); A. Owyoung, IEEE J. Quantum Electron. QE-9, 1064 (1973).

${ }^{11}$ T. W. Stinson, J. D. Litster, and N. A. Clark, J. Phys. (Paris) 33, 69 (1972).

${ }^{12}$ The scattering loss coefficient $\alpha /\left(T-T^{*}\right)$ is obtained from

$$
\left(\frac{\omega}{c}\right)^{4} \frac{V}{(4 \pi)^{2}} \int_{\Omega}\left\langle\delta \epsilon_{i f}^{2}(q)\right\rangle d \Omega
$$

with $\left\langle\delta \epsilon_{i f}^{2}(q)\right\rangle$ given by Eq. (15) of Ref. 11. One can easily show that $\alpha$ is proportional to $\omega^{4}(\Delta \chi)^{2} / a$. The values of $\alpha$ we measured for MBBA and EBBA at $6943 \AA$ are $0.1 \mathrm{~cm}^{-1}{ }^{\circ} \mathrm{K}$ and $0.06 \mathrm{~cm}^{-1}{ }^{\circ} \mathrm{K}$, respectively.

${ }^{13}$ I. Haller, H. A. Huggins, M. J. Freiser, Mol. Cryst. Liq. Cryst. 16, 53 (1972).

${ }^{14} \mathrm{~J}$. Prost and J. R. Lalanne, Phys. Rev. A $\underline{8}, 2090$ (1973). 